\title{
Matrix continued fraction representation of the Gauss hypergeometric function
}

\author{
S. Mennou ${ }^{1}$, S. Salhi ${ }^{2}$ and A. Kacha ${ }^{1}$ \\ ${ }^{1}$ Ibn Toufail University, Science faculty, Laboratory AMGNCA, Kenitra 14000, Morocco \\ ${ }^{2}$ Regional Center for Education and Training Professions P. B. 8, Errachidia, Morocco
}

Received: 13 June 2020, Accepted: 27 December 2020

Published online: 21 March 2021.

\begin{abstract}
The aim of this paper is to give some properties and results of continued fractions with matrix arguments. Then we give continued fraction expansion of the Gauss hypergeometric function. At the end, some numerical examples illustrating the theoretical results are discussed.
\end{abstract}

Keywords: Matrix continued fractions, Convergence criteria, Hypergeometric function.

\section{Introduction and motivation}

The theory of continued fractions has been a topic of great interest over the last two hundred years. The basic idea of this theory over real numbers is to give an approximation of various real numbers by the rational ones. A continued fraction is an expression obtained through an iterative process of representing a number as the sum of its integer part and the reciprocal of another number, then writing this other number as the sum of its integer part and another reciprocal, and so on. One of the main raisons why continued fractions are so useful in computation is that they often provide representation for transcendental functions that are much more generally valid than the classical representation by, say,the power series. Further; in the convergent case, the continued fractions expansions have the advantage that they converge more rapidly than other numerical algorithms.

Recently, the extension of continued fractions theory from real numbers to the matrix case has seen several developments and interesting applications (see [1,6,7]). Since calculations involving matrix valued functions with matrix arguments are feasible with large computers, it will be interesting attempt to develop such matrix theory. In this direction, and generally in a Banach space, few convergence results on noncommutative continued fraction are known $([4,5])$.

Two theorems are stated in [13], where Wynn reviews many aspects of the theory of continued fractions, whose elements do not commute under a multiplication law. In Banach space, extensions of Worpitsky's have been proven by Haydan [5] and Negoescu [11].

In [12], several convergence criteria on the noncommutative continued fractions whose arguments are $m \times m$ matrices of the forms $K\left(B_{n} / A_{n}\right)$ are given.

The Gaussian or ordinary hypergeometric function ${ }_{2} F_{1}(a, b ; c ; z)$ is a special function represented by the hypergeometric series, that includes many other special functions as specific or limiting cases. It is a solution of a second-order linear 
ordinary differential equation (ODE). Every second-order linear ODE with three regular singular points can be transformed into this equation. For systematic lists of some of the many published identities involving the hypergeometric function, see the reference works by Olde Daalhuis ([2,8]. There is no known system for organizing all of the identities; indeed, there is no known algorithm that can generate all identities, a number of different algorithms are known that generate different series of identities. The theory of the algorithmic discovery of identities remains an active research topic.

\section{Preliminary and notations}

Throughout this paper, we denote by $\mathscr{M}_{m}$ the set of mxm real (or complex) matrices endowed with the subordinate matrix infinity norm defined by,

$$
\forall A=\left(a_{i, j}\right) \in \mathscr{M}_{m},\|A\|=\max _{1 \leq i \leq m} \sum_{j=1}^{m}\left|a_{i, j}\right|
$$

This norm satisfies the inequality

$$
\|A B\| \leq\|A\|\|B\|
$$

Let $A \in \mathscr{M}_{m}, A$ is said to be positive semidefinite (resp. positive definite) if $A$ is symmetric and

$$
\forall x \in \mathbb{R}^{m},(A x, x) \geq 0\left(\text { resp. } \forall x \in \mathbb{R}^{m}, x \neq 0,(A x, x)>0\right)
$$

where $(.,$.$) denotes the standard scalar product of \mathbb{R}^{m}$.

For any $A, B \in \mathscr{M}_{m}$ with $B$ invertible, we write $A / B=B^{-1} A$, in particular, if $A=I$, where $I$ is the $m^{\text {th }}$ order identity matrix, then we write $I / B=B^{-1}$. It is clear that for any invertible matrix $C$, we have

$$
\frac{C A}{C B}=\frac{A}{B}
$$

Definition 1. Let $\left(A_{n}\right)_{n \geq 0},\left(B_{n}\right)_{n \geq 1}$ be two nonzero sequences of $\mathscr{M}_{m}$. The continued fraction of $\left(A_{n}\right)$ and $\left(B_{n}\right)$ denoted by $K\left(B_{n} / A_{n}\right)$ is the quantity

$$
A_{0}+\frac{B_{1}}{A_{1}+\frac{B_{2}}{A_{2+\ldots}}}=\left[A_{0} ; \frac{B_{1}}{A_{1}}, \frac{B_{2}}{A_{2}}, \ldots\right] .
$$

Sometimes, we use briefly the notation

$$
\left[A_{0} ; \frac{B_{k}}{A_{k}}\right]_{k=1}^{+\infty}
$$

or

$$
K\left(B_{n} / A_{n}\right)
$$

where

$$
\left[A_{0} ; \frac{B_{k}}{A_{k}}\right]_{k=1}^{n}=\left[A_{0} ; \frac{B_{1}}{A_{1}}, \frac{B_{2}}{A_{2}}, \ldots, \frac{B_{n}}{A_{n}}\right] .
$$

The fractions

$$
\frac{B_{n}}{A_{n}}
$$


and

$$
\frac{P_{n}}{Q_{n}}:=\left[A_{0} ; \frac{B_{k}}{A_{k}}\right]_{k=1}^{n}
$$

are called, respectively, the $n^{\text {th }}$ partial quotient and the $n^{\text {th }}$ convergent of the continued fraction $K\left(B_{n} / A_{n}\right)$.

When $B_{n}=I$ for all $n \geq 1$, then $K\left(I / A_{n}\right)$ is called an ordinary continued fraction. The following proposition gives an adequate method to calculate $K\left(B_{n} / A_{n}\right)$.

Proposition 1. The elements $\left(P_{n}\right)_{n \geq-1}$ and $\left(Q_{n}\right)_{n \geq-1}$ of the $n^{\text {th }}$ convergent of $K\left(B_{n} / A_{n}\right)$ are given by the relationships

$$
\left\{\begin{array}{l}
P_{-1}=I, P_{0}=A_{0} \\
Q_{-1}=0, \quad Q_{0}=I
\end{array}\right.
$$

and

$$
\left\{\begin{array}{c}
P_{n}=A_{n} P_{n-1}+B_{n} P_{n-2} \\
Q_{n}=A_{n} Q_{n-1}+B_{n} Q_{n-2}
\end{array}, n \geq 1 .\right.
$$

Proof. We prove it by induction.

The proof of the next Proposition is elementary and we left it to the reader.

Proposition 2. For any two matrices $C$ and $D$ with $C$ invertible, we have

$$
C\left[A_{0} ; \frac{B_{k}}{A_{k}}\right]_{k=1}^{n} D=\left[C A_{0} D ; \frac{B_{1} D}{A_{1} C^{-1}}, \frac{B_{2} C^{-1}}{A_{2}}, \frac{B_{k}}{A_{k}}\right]_{k=3}^{n} .
$$

The continued fraction $K\left(B_{n} / A_{n}\right)$ converges in $\mathscr{M}_{m}$ if the sequence $\left(F_{n}\right)=\left(\frac{P_{n}}{Q n}\right)=\left(Q_{n}^{-1} P_{n}\right)$ converges in $\mathscr{M}_{m}$ in the sense that there exists a matrix $F \in \mathscr{M}_{m}$ such that $\lim _{n \rightarrow+\infty}|| F_{n}-F||=0$. In the other case, we say that $K\left(B_{n} / A_{n}\right)$ is divergent. It is clear that

$$
\frac{P_{n}}{Q_{n}}=A_{0}+\sum_{i=1}^{n}\left(\frac{P_{i}}{Q_{i}}-\frac{P_{i-1}}{Q_{i-1}}\right)
$$

From (2), we see that the continued fraction $K\left(B_{n} / A_{n}\right)$ converges in $\mathscr{M}_{m}$ if and only if the series $\sum_{n=1}^{+\infty}\left(\frac{P_{n}}{Q_{n}}-\frac{P_{n-1}}{Q_{n-1}}\right)$ converge in $\mathscr{M}_{m}$.

Definition 2. Let $\left(A_{n}\right),\left(B_{n}\right),\left(C_{n}\right)$ and $\left(D_{n}\right)$ be four sequences of matrices. We say that the continued fractions $K\left(B_{n} / A_{n}\right)$ and $K\left(D_{n} / C_{n}\right)$ are equivalent if we have $F_{n}=G_{n}$ for all $n \geq 1$, where $F_{n}$ and $G_{n}$ are the $n^{\text {th }}$ convergent of $K\left(B_{n} / A_{n}\right)$ and $K\left(D_{n} / C_{n}\right)$ respectively.

The following lemma characterizes equivalence of continued fractions.

Lemma 1. [6]. Let $\left(r_{n}\right)$ be a non-zero sequence of real numbers. The continued fractions

$$
\left[a_{0} ; \frac{r_{1} b_{1}}{r_{1} a_{1}}, \frac{r_{2} r_{1} b_{2}}{r_{2} a_{2}}, \ldots, \frac{r_{n} r_{n-1} b_{n}}{r_{n} a_{n}}, \ldots\right] \text { and }\left[a_{0} ; \frac{b_{1}}{a_{1}}, \frac{b_{2}}{a_{2}}, \ldots, \frac{b_{n}}{a_{n}}, \ldots\right]
$$

are equivalent.

We also recall the following Lemma, from the development of a function given by the Taylor series, we give the development in continued fractions of the series that was established by Euler. 
Lemma 2. [7]. Let $f$ be a function with the Taylor series development is $f(x)=\sum_{n=0}^{+\infty} c_{n} x^{n}$ in $J \subset \mathbb{R}$. Then, the development in continued fraction of $f(x)$ is

$$
f(x)=\left[c_{0} ; \frac{c_{1} x}{1}, \frac{-c_{2} x}{c_{1}+c_{2} x}, \frac{-c_{1} c_{3} x}{c_{2}+c_{3} x}, \ldots \frac{-c_{n-2} c_{n} x}{c_{n-1}+c_{n} x}, \ldots\right]
$$

Remark. Let $\left(A_{n}\right)$ and $\left(B_{n}\right)$ be two sequences of $\mathscr{M}_{m}$, we notice that we can write the first convergents of the continued fraction $K\left(B_{n} / A_{n}\right)$ by:

$$
\begin{gathered}
F_{1}=A_{0}+A_{1}^{-1} B_{1}=A_{0}+\left(B_{1}^{-1} A_{1}\right)^{-1} . \\
F_{2}=A_{0}+\left(A_{1}+A_{2}^{-1} B_{2}\right)^{-1} B_{1}=A_{0}+\left(B_{1}^{-1} A_{1}+\left(B_{2}^{-1} A_{2} B_{1}\right)^{-1}\right)^{-1} .
\end{gathered}
$$

If we put, $A_{1}^{*}=B_{1}^{-1} A_{1}$ and $A_{2}^{*}=B_{2}^{-1} A_{2} B_{1}$, we have

$$
F_{1}=A_{0}+\frac{I}{A_{1}^{*}}, F_{2}=A_{0}+\frac{I}{A_{1}^{*}+\frac{I}{A_{2}^{*}}}
$$

Generally, we prove by a recurrence that if we put for all $k \geq 1$,

$$
A_{2 k}^{*}=\left(B_{2 k} \ldots B_{2}\right)^{-1} A_{2 k} B_{2 k-1} \ldots B_{1}
$$

and

$$
A_{2 k+1}^{*}=\left(B_{2 k+1} \ldots B_{1}\right)^{-1} A_{2 k+1} B_{2 k} \ldots B_{2},
$$

then the continued fractions $A_{0}+K\left(B_{n} / A_{n}\right)$ and $A_{0}+K\left(I / A_{n}^{*}\right)$ are equivalent.

We need to present the following proposition:

Proposition 3. ([12]) Let $\left[A_{0} ; \frac{B_{k}}{A_{k}}\right]_{k=1}^{+\infty}$ be a given continued fraction. Then

$$
\frac{P_{n}}{Q_{n}}=\left[A_{0} ; \frac{B_{k}}{A_{k}}\right]_{k=1}^{n}=\left[A_{0} ; \frac{X_{k} B_{k} X_{k-2}^{-1}}{X_{k} A_{k} X_{k-1}^{-1}}\right]_{k=1}^{n},
$$

where $X_{-1}=X_{0}=I$ and $X_{1}, X_{2}, \ldots, X_{n}$ are arbitrary invertible matrices.

To end this section, we give the following Proposition.

Proposition 4. [11]. If the function $f(x)$ can be expanded in a power series in the circle $\left|x-x_{0}\right|<r$ by

$$
f(x)=\sum_{k=0}^{+\infty} a_{k}\left(x-x_{0}\right)^{k}
$$

then this expansion remains valid when the scalar argument $x$ is replaced by a matrix $A$ whose characteristic values lie within the circle of convergence. 


\section{Main results}

\subsection{The real case}

The Gauss hypergeometric function ${ }_{2} F_{1}$ is defined in $[10]$ as follows for $a, b, x \in \mathbb{R}, c \in \mathbb{R} / \mathbb{Z}^{-}$such that $|x|<1$, by

$$
{ }_{2} F_{1}(a, b ; c ; x)=\sum_{n=0}^{+\infty} \frac{(a)_{n}(b)_{n}}{(c)_{n}} \frac{x^{n}}{n !}
$$

where, for some parameter $\mu$, the Pochhammer symbol $(\mu)_{j}$ is defined as $(\mu)_{0}=1 ;(\mu)_{j}=\mu(\mu+1) \ldots(\mu+j-1)$, $j=1,2, \ldots$

As explained in [6], the Gauss hypergeometric function satisfies the differential equation

$$
x(1-x) \frac{d^{2} y}{d x^{2}}+[c-(a+b+1) x] \frac{d y}{d x}-a b y=0
$$

The equation (4) has three regular singular points at $x=0, x=1$ and $x=\infty$.

If $a \in \mathbb{Z}^{-}$or $b \in \mathbb{Z}^{-}$, the function ${ }_{2} F_{1}(a, b ; c ; x)$ is reduced to polynomial in $x$.

We recall that many elementary and special functions can be expressed in terms of hypergeometric functions.

\section{Example 1.}

$$
\begin{gathered}
{ }_{2} F_{1}(1,1 ; 2 ; x)=-x^{-1} \ln (1-x), \\
{ }_{2} F_{1}\left(1 / 2,1 ; 3 / 2 ; x^{2}\right)=\frac{1}{2 x} \ln \left(\frac{1+x}{1-x}\right), \\
{ }_{2} F_{1}\left(1 / 2,1 ; 3 / 2 ; x^{2}\right)=x^{-1} \arctan (x), \\
\frac{{ }_{2} F_{1}\left(1 / 2,-1 / 2 ; 1 / 2 ; x^{2}\right)}{{ }_{2} F_{1}\left(1 / 2,1 / 2 ; 3 / 2 ; x^{2}\right)}=\frac{x \sqrt{1-x^{2}}}{\arcsin (x)}, \\
\frac{x^{a}{ }_{2} F_{1}(a, 1-b ; a+1 ; x)=B_{x}(a, b)=\int_{0}^{x} t^{a-1}(1-t)^{b-1} d t,}{}
\end{gathered}
$$

where $a, b \in \mathbb{R}^{+}, 0 \leq x \leq 1$ and $B_{x}(a, b)$ is the incomplete beta function.

Theorem 1. Let $x$ be a real number $|x|<1$, the continued fraction representation of the Gauss hypergeometric function is

$$
{ }_{2} F_{1}(a, b ; c ; x)=\left[1 ; \frac{a b x}{c}, \frac{-(n-1)(c+n-2)(a+n-1)(b+n-1) x}{n(c+n-1)+(a+n-1)(b+n-1) x}\right]_{n=2}^{+\infty}
$$

Proof. We apply Lemma 2.6 to the function: ${ }_{2} F_{1}(a, b ; c ; x)=\sum_{n=0}^{+\infty} c_{n} x^{n}$ by putting $c_{n}=\frac{(a)_{n}(b)_{n}}{(c)_{n} n !}$. So, we have

$$
c_{0}=1, \frac{c_{1} x}{1}=\frac{\frac{a b}{c} x}{1}, \frac{-c_{2} x}{c_{1}+c_{2} x}=\frac{\frac{-(a)_{2}(b)_{2}}{2(c)_{2}} x}{\frac{a b}{c}+\frac{(a)_{2}(b)_{2}}{2(c)_{2}} x} .
$$

For $n \geq 3$, we get

$$
c_{n-2} c_{n} x=\frac{(a)_{n-2}(b)_{n-2}(a)_{n}(b)_{n}}{(c)_{n-2}(n-2) !(c)_{n} n !} x
$$

Furthermore, we have

$$
c_{n-1}+c_{n} x=\frac{(a)_{n-1}(b)_{n-1}}{(c)_{n-1}(n-1) !}+\frac{(a)_{n}(b)_{n}}{(c)_{n} n !} x
$$


Then, we obtain

$$
\frac{-c_{n-2} \cdot c_{n} x}{c_{n-1}+c_{n} x}=\frac{-\frac{(a)_{n-2}(b)_{n-2}(a)_{n}(b)_{n}}{(c)_{n-2}(n-2) !(c)_{n} n !} x}{\frac{(a)_{n-1}(b)_{n-1}}{(c)_{n-1}(n-1) !}+\frac{(a)_{n}(b)_{n}}{(c)_{n} n !} x}
$$

Therefore, the continued fraction expansion of ${ }_{2} F_{1}(a, b ; c ; x)$ is

$$
{ }_{2} F_{1}(a, b ; c ; x)=\left[1 ; \frac{\frac{a b}{c} x}{1}, \frac{\frac{-(a)_{2}(b)_{2}}{2(c)_{2}} x}{\frac{a b}{c}+\frac{(a)_{2}(b)_{2}}{2(c)_{2}} x}, \frac{-\frac{(a)_{n-2}(b)_{n-2}(a)_{n}(b)_{n}}{(c)_{n-2}(n-2) !(c)_{n} n !} x}{\frac{(a)_{n-1}(b)_{n-1}}{(c)_{n-1}(n-1) !}+\frac{(a)_{n}(b)_{n}}{(c)_{n} n !} x}\right]_{n=3}^{+\infty} .
$$

In order to simplify the above equality, let us define the sequence $\left(r_{n}\right)_{n \geq 1}$ by

$$
\left\{\begin{array}{l}
r_{1}=c \\
r_{n}=\frac{(c)_{n} n !}{(a)_{n-1}(b)_{n-1}} \text { for } n \geq 2
\end{array}\right.
$$

Then, we have

$$
\left\{\begin{array}{l}
\frac{r_{1} b_{1}}{r_{1} a_{1}}=\frac{a b x}{c} \\
\frac{r_{1} r_{2} b_{2}}{r_{2} a_{2}}=\frac{-c(a+1)(b+1) x}{2(c+1)+(a+1)(b+1) x}, \\
\frac{r_{n} r_{n-1} b_{n}}{r_{n} a_{n}}=\frac{-(n-1)(c+n-2)(a+n-1)(b+n-1) x}{n(c+n-1)+(a+n-1)(b+n-1) x} \text { for } n \geq 3 .
\end{array}\right.
$$

By applying the result of Lemma 2.5 to the sequence $\left(r_{n}\right)_{n \geq 1}$, we obtain

$$
{ }_{2} F_{1}(a, b ; c ; x)=\left[1 ; \frac{a b x}{c}, \frac{-(n-1)(c+n-2)(a+n-1)(b+n-1) x}{n(c+n-1)+(a+n-1)(b+n-1) x}\right]_{n=2}^{+\infty}
$$

and the proof is complete.

Corollary 1. Let $x$ be a real number $|x|<1$. From Theorem 3.3, Lemma 2.5 and by appropriate transformations, we deduce the continued fraction representations of the special functions which we have seen in the above examples.

(i)

(ii)

$$
\ln (1-x)=\left[-x ; \frac{-x^{2}}{2}, \frac{-2 x}{1+x}, \frac{\frac{-n}{n-1} x}{1+x}\right]_{n=3}^{+\infty}
$$

$$
\arctan (x)=\left[x ; \frac{x^{3}}{1}, \frac{3 x^{2}}{1-3 x^{2}}, \frac{(n-1 / 2)(n-5 / 2) x^{2}}{(n-3 / 2)-(n-1 / 2) x^{2}}\right]_{n=3}^{+\infty}
$$

\subsection{The matrix case}

Definition 3. Let $A$ be a matrix in $\mathscr{M}_{m}$, such that $\|A\|<1$, we define the Gauss hypergeometric function of a matrix $A$ by the expression

$$
{ }_{2} F_{1}(a, b ; c ; A)=\sum_{n=0}^{+\infty} \frac{(a)_{n}(b)_{n}}{(c)_{n} n !} A^{n} .
$$

Theorem 2. Let $A \in \mathscr{M}_{m}$ be a positive definite matrix such that $\|A\|<1$. The representation continued fraction of the Gauss hypergeometric function ${ }_{2} F_{1}(a, b ; c ; A)$ is

$$
{ }_{2} F_{1}(a, b ; c ; A)=\left[I ; \frac{a b A}{c I}, \frac{-(n-1)(c+n-2)(a+n-1)(b+n-1) A}{n(c+n-1) I+(a+n-1)(b+n-1) A}\right]_{n=2}^{+\infty} .
$$


Proof. Since $A$ is a positive definite matrix, then there exists an invertible matrix $X$ such that $A=X D X^{-1}$ where $D=\operatorname{diag}\left(\lambda_{1}, \lambda_{2}, \ldots, \lambda_{m}\right)$ and $\lambda_{i}>0$ for all $1 \leq i \leq m$. As the function $F={ }_{2} F_{1}(a, b ; c ;)$ is analytic in a domain $J=]-1,1[$ then

$$
F(A)=F\left(X D X^{-1}\right)=X F\left(\operatorname{diag}\left(\lambda_{1}, \lambda_{2}, \ldots, \lambda_{m}\right)\right) X^{-1}=X \operatorname{diag}\left(F\left(\lambda_{1}\right), F\left(\lambda_{2}\right), \ldots, F\left(\lambda_{m}\right)\right) X^{-1}
$$

Let us define the sequences $\left(P_{n}\right)$ and $\left(Q_{n}\right)$ by

$$
\begin{gathered}
\qquad\left\{\begin{array}{l}
P_{-1}=I, P_{0}=I, P_{1}=c I+a b A, \\
Q_{-1}=0, Q_{0}=I, Q_{1}=c I,
\end{array} \text { and for } n \geq 2,\right. \\
\left\{\begin{array}{l}
\left.P_{n}=(n(c+n-1) I+(a+n-1)(b+n-1) A) P_{n-1}-(n-1)(c+n-2)(a+n-1)(b+n-1) A\right) P_{n-2}, \\
\left.Q_{n}=(n(c+n-1) I+(a+n-1)(b+n-1) A) Q_{n-1}-(n-1)(c+n-2)(a+n-1)(b+n-1) A\right) Q_{n-2} .
\end{array}\right.
\end{gathered}
$$

We see that $P_{n}$ and $Q_{n}$ are diagonal matrices. By setting $P_{n}=\operatorname{diag}\left(p_{n}^{1}, \ldots, p_{n}^{m}\right)$, and $Q_{n}=\operatorname{diag}\left(q_{n}^{1}, \ldots, q_{n}^{m}\right)$, We obtain for each $i, 1 \leq i \leq m$,

$$
\begin{gathered}
\qquad\left\{\begin{array}{l}
p_{-1}^{i}=1, p_{0}^{i}=1, p_{1}^{i}=c+a b \lambda_{i}, \quad \text { and for } n \geq 2, \\
q_{-1}^{i}=0, q_{0}^{i}=1, q_{1}^{i}=c,
\end{array}\right. \\
\left\{\begin{array}{l}
p_{n}^{i}=\left(n(c+n-1)+(a+n-1)\left(b+n-1 \lambda_{i}\right) p_{n-1}^{i}+\left(-(n-1)(c+n-2)(a+n-1)(b+n-1) \lambda_{i}\right) p_{n-2}^{i},\right. \\
q_{n}^{i}=\left(n(c+n-1) I+(a+n-1)(b+n-1) \lambda_{i}\right) q_{n-1}^{i}+\left(-(n-1)(c+n-2)(a+n-1)(b+n-1) \lambda_{i}\right) q_{n-2}^{i} .
\end{array}\right.
\end{gathered}
$$

By Theorem 3.1 the convergent $\left(p_{n}^{i} / q_{n}^{i}\right)$ converges to $F\left(\lambda_{i}\right)$. It follows that $P_{n} / Q_{n}$ converges to the matrix $\operatorname{diag}\left(F\left(\lambda_{1}\right), F\left(\lambda_{2}\right), \ldots, F\left(\lambda_{m}\right)\right)$, so that

$$
F(D)=\left[I ; \frac{a b D}{c I}, \frac{-(n-1)(c+n-2)(a+n-1)(b+n-1) D}{n(c+n-1) I+(a+n-1)(b+n-1) D}\right]_{n=2}^{+\infty}
$$

then

$$
\begin{aligned}
F(A) & =X\left[I ; \frac{a b D}{c I}, \frac{-(n-1)(c+n-2)(a+n-1)(b+n-1) D}{n(c+n-1) I+(a+n-1)(b+n-1) D}\right]_{n=3}^{+\infty} X^{-1} \\
& =\left[I ; \frac{a b D X^{-1}}{c X^{-1}}, \frac{-(n-1)(c+n-2)(a+n-1)(b+n-1) D}{n(c+n-1) I+(a+n-1)(b+n-1) D}\right]_{n=2}^{+\infty}
\end{aligned}
$$

Let us define the sequence $\left(X_{n}\right)_{n \geq-1}$ by

$$
\left\{\begin{array}{l}
X_{-1}=X_{0}=I \\
X_{n}=X, \text { for } n \geq 1
\end{array}\right.
$$


By applying the result of proposition 2.7 to the sequence $\left(X_{n}\right)_{n \geq-1}$, we obtain

$$
\left\{\begin{array}{l}
\frac{X_{1} a b D X^{-1} X_{-1}^{-1}}{X_{1} c X^{-1} X_{0}^{-1}}=\frac{a b A}{c I} \\
\frac{X_{2}(-c(a+1)(b+1) D) X_{1}^{-1}}{X_{2}(2(c+1) I+(a+1)(b+1) D) X_{0}^{-1}} \frac{-c(a+1)(b+1) A}{2(c+1) I+(a+1)(b+1) A} \\
\frac{X_{n} b_{n} D X_{n-2}^{-1}}{X_{n} a_{n} D X_{n-1}^{-1}}=\frac{b_{n} A}{a_{n} A} .
\end{array}\right.
$$

where $b_{n}=-(n-1)(c+n-2)(a+n-1)(b+n-1)$ and $a_{n}=n(c+n-1) I+(a+n-1)(b+n-1)$ for $n \geq 3$. Which finish the proof of Theorem 3.4.

\section{Numerical applications}

This section will provide some numerical data to illustrate the preceding results. The focus will be on two cases.

\subsection{Real case}

The following table clarifies the differences between ${ }_{2} F_{1}(x)$ and its first convergents when Applying Theorem 3.4

\begin{tabular}{|c|c|c|c|c|c|}
\hline $\mathrm{x}$ & $\frac{\left({ }_{2} F_{1}-F_{1}\right)(x)}{{ }_{2} F_{1}(x)}$ & $\frac{\left({ }_{2} F_{1}-F_{2}\right)(x)}{{ }_{2} F_{1}(x)}$ & $\frac{\left({ }_{2} F_{1}-F_{3}\right)(x)}{{ }_{2} F_{1}(x)}$ & $\frac{\left.{ }_{2} F_{1}-F_{4}\right)(x)}{{ }_{2} F_{1}(x)}$ & $\frac{\left.{ }_{2} F_{1}-F_{5}\right)(x)}{{ }_{2} F_{1}(x)}$ \\
\hline 0.005 & $0.5009625484 \mathrm{e}-5$ & $0.1796995989 \mathrm{e}-7$ & 0 & 0 & 0 \\
\hline 0.05 & $0.5098196233 \mathrm{e}-3$ & $0.1826707577 \mathrm{e}-4$ & $0.7117680889 \mathrm{e}-6$ & $0.2949315285 \mathrm{e}-7$ & $0.1966210190 \mathrm{e}-8$ \\
\hline 0.1 & $0.2081107059 \mathrm{e}-2$ & $0.1496511363 \mathrm{e}-3$ & $0.1169013697 \mathrm{e}-4$ & $0.9589678655 \mathrm{e}-6$ & $0.8112114874 \mathrm{e}-7$ \\
\hline 0.2 & $0.8694693918 \mathrm{e}-2$ & $0.1259904125 \mathrm{e}-2$ & $0.1977914303 \mathrm{e}-3$ & $0.3257367278 \mathrm{e}-4$ & $0.5538918396 \mathrm{e}-5$ \\
\hline 0.3 & $0.2052794626 \mathrm{e}-1$ & $0.4500221749 \mathrm{e}-2$ & $0.1065709225 \mathrm{e}-2$ & $0.2643229997 \mathrm{e}-3$ & $0.6761918887 \mathrm{e}-4$ \\
\hline 0.4 & $0.3851816176 \mathrm{e}-1$ & $0.1137043926 \mathrm{e}-1$ & $0.3613947237 \mathrm{e}-2$ & $0.1200815877 \mathrm{e}-2$ & $0.4110640272 \mathrm{e}-3$ \\
\hline 0.5 & $0.6400881084 \mathrm{e}-1$ & $0.2389490275 \mathrm{e}-1$ & $0.9568506885 \mathrm{e}-2$ & $0.3997131117 \mathrm{e}-2$ & $0.1717931867 \mathrm{e}-2$ \\
\hline 0.6 & $0.9906047842 \mathrm{e}-1$ & $0.4500410712 \mathrm{e}-1$ & $0.2183709075 \mathrm{e}-1$ & $0.1102581649 \mathrm{e}-1$ & $0.5718463603 \mathrm{e}-2$ \\
\hline 0.7 & 0.1471603053 & $0.7939412418 \mathrm{e}-1$ & $0.4551103360 \mathrm{e}-1$ & $0.2706357301 \mathrm{e}-1$ & $0.1649820925 \mathrm{e}-1$ \\
\hline 0.8 & 0.2152166332 & 0.1359122088 & $0.9059539494 \mathrm{e}-1$ & $0.6239826632 \mathrm{e}-1$ & $0.4394196386 \mathrm{e}-1$ \\
\hline 0.9 & 0.3217901569 & 0.2372747765 & 0.1829434606 & 0.1449115394 & 0.1169062156 \\
\hline
\end{tabular}

We can clearly see that $F_{5}$ is approximately the exact value of ${ }_{2} F_{1}(x)$.

\subsection{Matrix case}

Example 2. Let $A$ be a $2 \times 2$ matrix, such that

$$
A=\left(\begin{array}{cc}
\frac{3}{64} & \frac{1}{32} \\
\frac{1}{32} & \frac{3}{64}
\end{array}\right)
$$

A is a positive definite matrix such that $\|A\|=5 / 64<1$.

We calculate the difference between ${ }_{2} F_{1}$ and the first five convergents, by using the expansion of Theorem 3.4, such that $a=\frac{1}{2}, b=1$ and $c=\frac{3}{2}$, we obtain the following results

$$
{ }_{2} F_{1}-F_{1}=\left(\begin{array}{l}
0.671312531689949310 e-30.621932741383298890 e-3 \\
0.621932741383298890 e-30.671312531689949310 e-3
\end{array}\right) .
$$




$$
\begin{aligned}
& { }_{2} F_{1}-F_{2}=\left(\begin{array}{ll}
0.365469066898604922 e-4 & 0.359952413832992368 e-4 \\
0.359952413832992368 e-4 & 0.3654690668998604922 e-4
\end{array}\right) . \\
& { }_{2} F_{1}-F_{3}=\left(\begin{array}{ll}
0.221463129990162600 e-5 & 0.220792274490018425 e-5 \\
0.220792274490018425 e-5 & 0.221463129990162600 e-5
\end{array}\right) . \\
& { }_{2} F_{1}-F_{4}=\left(\begin{array}{ll}
0.141714209922483292 e-6 & 0.141628392699363892 e- \\
0.141628392699363892 e-0 & 0.141714209922483292 e-6
\end{array}\right) . \\
& { }_{2} F_{1}-F_{5}=\left(\begin{array}{ll}
0.938174005149505774 e-8 & 0.938058710049649580 e-8 \\
0.938058710049649580 e-8 & 0.938174005149505774 e-8
\end{array}\right) .
\end{aligned}
$$

Example 3. Let $A$ be a $3 \times 3$ matrix, such that

$$
A=\left(\begin{array}{ccc}
\frac{1}{25} & \frac{1}{50} & \frac{3}{100} \\
\frac{1}{50} & \frac{1}{25} & \frac{1}{50} \\
\frac{3}{100} & \frac{1}{50} & \frac{1}{25}
\end{array}\right)
$$

we verify that $\mathrm{A}$ is a positive definite matrix and $\|A\|=9 / 100<1$.

We calculate the difference between ${ }_{2} F_{1}$ and the first five convergents, by using the expansion of Theorem 3.4, such that $a=\frac{1}{2}, b=1$ and $c=\frac{1}{2}$, we obtain the following results

$$
\begin{gathered}
{ }_{2} F_{1}-F_{1}=\left(\begin{array}{lll}
0.316709697875605656 e-2 & 0.242152466367712843 e-2 & 0.306608687774607288 e-2 \\
0.242152466367712843 e-2 & 0.260089686098652351 e-2 & 0.242152466367712843 e-2 \\
0.306608687774607288 e-2 & 0.242152466367712843 e-2 & 0.316709697875605656 e-2
\end{array}\right) . \\
{ }_{2} F_{1}-F_{2}=\left(\begin{array}{lll}
0.230969787560209028 e-4 & 0.221524663677127865 e-3 & 0.266086877746068984 e-3 \\
0.221524663677127865 e-3 & 0.200896860986565784 e-3 & 0.221524663677127865 e-3 \\
0.266086877746068984 e-3 & 0.221524663677127865 e-3 & 0.230969787560209028 e-4
\end{array}\right) . \\
{ }_{2} F_{1}-F_{3}=\left(\begin{array}{lll}
0.419300593001992184 e-5 & 0.195246636771305438 e-4 & 0.230868777460688235 e-4 \\
0.195246636771305438 e-4 & 0.168968609866038122 e-4 & 0.195246636771305438 e-4, \\
0.230868777460688235 e-4 & 0.195246636771305438 e-4 & 0.419300593001992184 e-5
\end{array}\right) . \\
{ }_{2} F_{1}-F_{4}=\left(\begin{array}{lll}
0.200697875607502852 e-5 & 0.170466367712882039 e-5 & 0.200687774606911340 e-5 \\
0.170466367712882039 e-5 & 0.145686098651509610 e-5 & 0.170466367712882039 e-5 \\
0.200687774606911340 e-5 & 0.170466367712882039 e-5 & 0.200697875607502852 e-5
\end{array}\right) . \\
{ }_{2} F_{1}-F_{5}=\left(\begin{array}{lll}
0.174578756118037860 e-6 & 0.148463677129839768 e-6 & 0.174577746071824526 e-6 \\
0.148463677129839768 e-6 & 0.126460986571430568 e-6 & 0.148463677129839768 e-6 \\
0.174577746071824526 e-6 & 0.148463677129839768 e-6 & 0.174578756118037860 e-6
\end{array}\right) .
\end{gathered}
$$


In the examples above, we can clearly see that $F_{5}$ is approximately the exact value of ${ }_{2} F_{1}(A)$.

Moreover when $n=16$ we will have ${ }_{2} F_{1}(A)-F_{16} \simeq 0$. This shows the importance of the continued fractions approach.

\section{Competing interests}

The authors declare that they have no competing interests.

\section{Authors' contributions}

All authors have contributed to all parts of the article. All authors read and approved the final manuscript.

\section{References}

[1] A. Cuyt, V.B. Petersen, Handbook of continued fractions for special functions, Springer (2007).

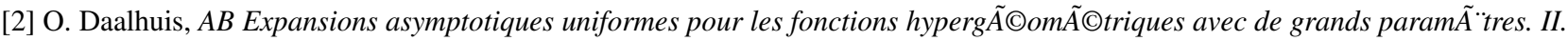
Anal. Appl. (Singapour) 1 (2003), no. 1, 121-128.

[3] F. R. Gantmacher, The Theory of Matrices, Vol. I. Chelsa, New York, Elsevier Science Publishers, (1992).

[4] V. Gen, H. Golub and C. F.Van Loan, Matrix computations, Johns Hopking University Press, Baltimore, MD, USA, third edition (1996).

[5] T. L. Hayden, Continued fractions in Banach spaces, Rocky Mtn. J.Math., 4 (1974), pp. 367-369.

[6] W. B. Jon and W. J. Thron, Continued fractions: analytic theory and applications, Addisson-Wesley, Encyclopedia of Mathematics and its Applications, vol. 11, London, Amsterdam, Sydney, Tokyo (1980).

[7] A. N. Khovanski, The applications of continued fractions and their Generalisation to problemes in approximation theory, 1963, Noordhoff, Groningen, The Netherlands (chap2).

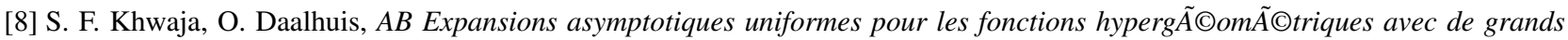
param A“tres IV Analysis and Applications 12 (2014), no. 6, 667-710.

[9] L. Lorentzen, H. Wadeland, Continued fractions with applications, Elsevier Science Publishers, 1992.

[10] Y. L. Luke,The Special Functions and Their Approximations, Volume I, Academic Press, 1969.

[11] N. Negoescu, Convergence theorems on noncommutative continued fractions, Rev. Anal. Numér. Theorie Approx., 5 (1977), pp. 165-180.

[12] M. Raissouli, A. Kacha , Convergence of matrix continued fractions. Linear Algebra Appl. 320 (2000), 115-129.

[13] P. Wynn, One some recent developments in the theory of continued fractions, SIAM J. Numer. Anal. 1 (1964), pp. 177-197. 